\title{
EVOLUTIONARY EFFECTS IN CONTACT BINARIES ${ }^{+}$
}

\author{
H.Mauder \\ Astronomisches Institut \\ Universität Tübingen
}

The structure and evolution of contact binaries is an extremely difficult problem in theoretical astrophysics. Several attempts have been made to set up a satisfactory model, see e.g. Lucy,1976, Flannery, 1976, Shu, Lubow and Anderson, 1976, Robertson and Eggleton, 1977. Rahunen and Vilhu, 1977 and others. There are, roughly, two lines of sight: Shu, Lubow and Anderson postulate the existence of a contact discontinuity in one of the two components of a contact binary (DSC Theory); in this case age zero models in thermal equilibrium are possible. The other authors find that unevolved contact binaries cannot achieve thermal equilibrium; therefore, relaxation oscillations on a thermal time scale are expected (TRO Theory). For a detailed discussion of the different effects see Lucy and Wilson,1979.

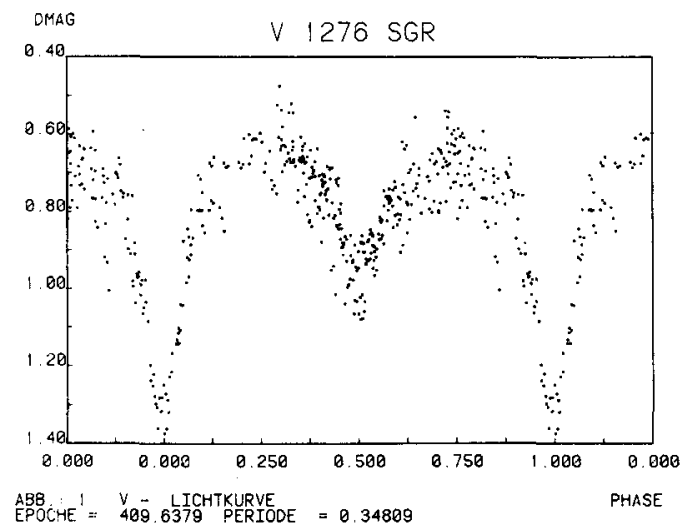

Figure 1:

$\mathrm{V}$ light curve of V1276 Sgr

+ Based on observations obtained at ESO/La Silla 
Though W UMa systems are very numerous, only a limited number has been studied in detail. It is necessary, therefore, to look for systems which might eventually show effects that are predicted or excluded by one of the theories, at least in their present form. We have started an observational campaign concentrating on stars with periods below 0.5 days and minima of unequal depths. One example is RW Dor with a period of 0.285 . This is an A-type system with a deep common envelope, a mass ratio of 0.38 and spectral type $\mathrm{KO}$. In secondary minimum the system is $0.16 \mathrm{mag}$ brighter than in primary minimum. Systems like RW Dor are difficult to understand in DSC theory. Another example is V 1276 Sgr with $\mathrm{P}=0,348$, spectral type $\mathrm{K} 3$ and mass ratio about 0.6 . V $1276 \mathrm{Sgr}$ is remarkably active with large intrinsic scatter in the light curve, see fig.1. For the moment it is not possible, therefore, to decide, whether the two components are in contact or not. The difference in the depths of the minima is $0.45 \mathrm{mag}$ in this case. No emissior lines are seen in the spectra, the masses are about 0.7 and 0.4 solar masses. Additional observations are planned for a better definition of the light curve.

$A$ very striking system we found is FT Lup = BV 851. The period is $P=0.470$, spectral type $F 1$ and mass ratio about 0.4 . The difference in the minima is $0.6 \mathrm{mag}$, primary minimum is due to an annular eclipse. The light curve is well defined, see fig.2, and a preliminary solution shows that the two components are just in contact. The masses of the two components are about $2 \mathrm{M}_{0}$ and $0.8 \mathrm{M}_{\odot}$.

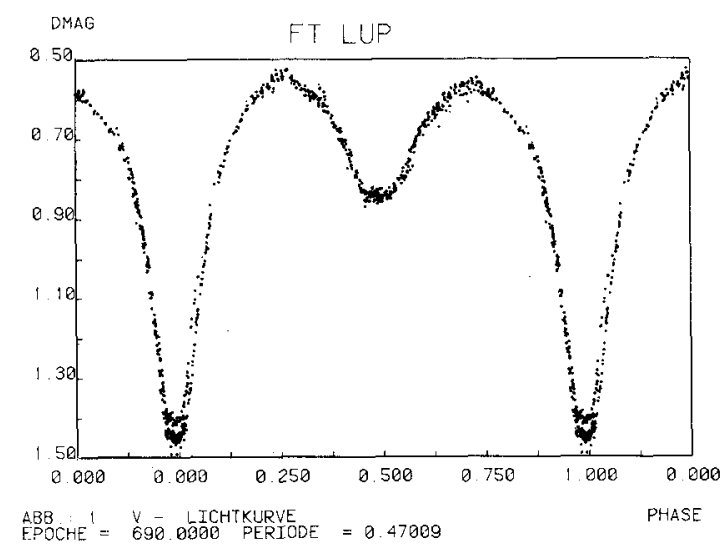

Figure 2:

$\mathrm{V}$ light curve of FT Iup

No peculiarities are seen in the spectra. With radii of about $1.65 \mathrm{R}_{\odot}$ and $1.06 \mathrm{R}_{\odot}$ and $\log \mathrm{T}_{\mathrm{e}}=3.87, \log \mathrm{T}_{\mathrm{e}, 2}=3.71$, the two componênts are cleârly not nombl zero age main'sequence objects. The two stars show the typical properties of the components of W UMa systems, namely too small radius and too low temperature of the primary star, too large radius and too high temperature for 
the secondary, as compared with zero age main sequence objects of the same mass. The only difference against $W$ UVa systems is the high difference in the surface temperatures between the two components.

The period of FT Lup has shortened remarkably within the last 80 years. In figure 3 the $\mathrm{O}-\mathrm{C}$ diagram is shown, according to the results of Bauernfeind, 1968 and Strohmeier,1967. In addition, the O-C values of the six photoelectric minima from March and June 1981 are indicated as a cross. From the 1981 p.e. minima a period of $0.4700730 \pm 0.0000015$ is derived. The period decrease can be interpreted as mass transfer from the primary to the secondary at a rate of more than $3^{*} 10^{-} \mathrm{N}_{\mathrm{O}}$ per year.

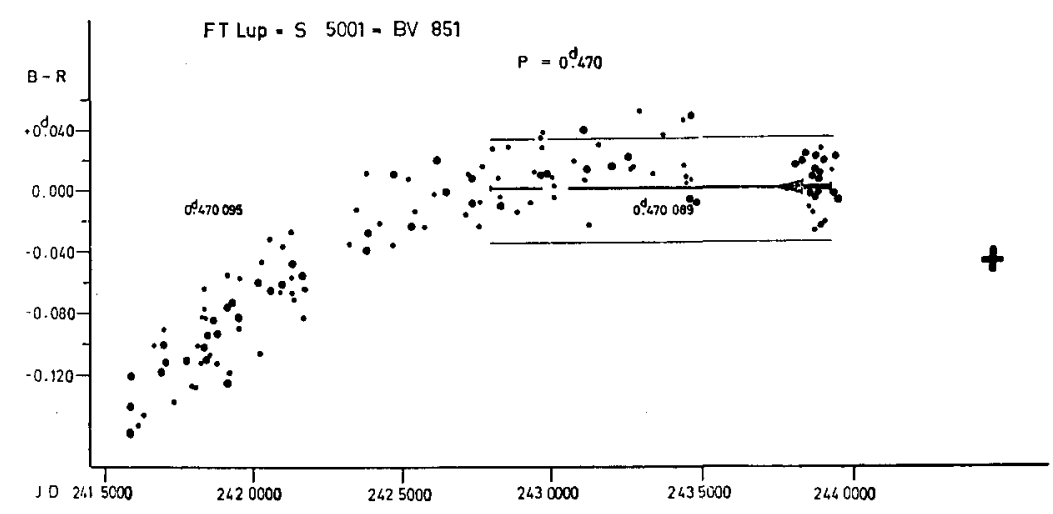

Figure 3: O-C diagram of pg minima of FT Lup. The cross indicates the $\mathrm{O}-\mathrm{C}$ of the $1981 \mathrm{p} . \mathrm{e}$. minima

It seems to be obvious that FT Lup is in a phase where the evolution towards the normal W UMa stage is at its very beginning. The degree of contact is not yet sufficient to build up a common envelope with constant specific entropy, but already strong enough to produce the deviations from the zero age main sequence. A comparison of the position of the components of FT Lup in the $\log g-\log \mathrm{T}$. diagrams of Hejlesen, 1980, with the possible evolutionary tracks ${ }^{e}$ of stars of equal mass, taking into account the possible alterations of $R$ and $T$, indicates a remarkably high content of heavy elements, $\mathrm{Z}$ about 0.84 , and an age of probably less than $5 \cdot 10^{8}$ years for FT Lup. The properties of this system, therefore, seem to be of special importance for the future development of DSC and TRO theories. 
References:

H.Bauernfeind,1968,Veröff.Bamberg 8 ,Nr. 81

B.P.Flannery, 1976, Ap.J.205, 217

P.M.Hejlesen, 1980,Astr. \&Astrophys.Suppl. 39, 347

L.B.Lucy , 1976, Ap.J. 205,208

L.B. Lucy and R.E.Wilson, 1979,Ap.J.231,502

T.Rahunen and Q.Vilhu,1977,Astr.\&Astrophys.56,99

J.A.Robertson and P.P.Eggleton,1977,MNRAS 175,279

F.M.Shu,S.H.Lubow and L.Anderson, 1976,Ap.J.209, 536

W.Strohmeier, 1967 ,IBVS 184 